\title{
The lower tail of the random minimum spanning tree
}

\author{
Abraham D. Flaxman \\ Microsoft Research \\ Redmond, WA, USA \\ abie@microsoft.com
}

Submitted: Sep 11, 2006; Accepted: Dec 15, 2006; Published: Jan 17, 2007

Mathematics Subject Classifications: 05C80, 60C05

\begin{abstract}
Consider a complete graph $K_{n}$ where the edges have costs given by independent random variables, each distributed uniformly between 0 and 1 . The cost of the minimum spanning tree in this graph is a random variable which has been the subject of much study. This note considers the large deviation probability of this random variable. Previous work has shown that the log-probability of deviation by $\varepsilon$ is $-\Omega(n)$, and that for the log-probability of $Z$ exceeding $\zeta(3)$ this bound is correct; $\log \operatorname{Pr}[Z \geq \zeta(3)+\varepsilon]=-\Theta(n)$. The purpose of this note is to provide a simple proof that the scaling of the lower tail is also linear, $\log \operatorname{Pr}[Z \leq \zeta(3)-\varepsilon]=-\Theta(n)$.
\end{abstract}

\section{Introduction}

If the edge costs of the complete graph $K_{n}$ are independent random variables, each uniformly distributed between 0 and 1 , then the cost of a minimum spanning tree is a random variable which has expectation asymptotically equal to $\zeta(3)=\sum_{i=1}^{\infty} i^{-3}$ [6]. Furthermore, after an appropriate rescaling, this random variable converges in distribution to a Gaussian distribution with an explicitly known variance of about 1.6857 [8]. This note considers the large deviation probability of this random variable, denoted $Z_{n}$.

In [9], as an example application of Talagrand's Inequality, McDiarmid shows that $Z_{n}$ satisfies an exponential tail inequality of the form

$$
\operatorname{Pr}\left[\left|Z_{n}-\zeta(3)\right| \geq \varepsilon\right] \leq e^{-C_{\varepsilon} n} .
$$

(See also [4] for an alternative approach with additional details). Simple considerations show that for the log-probability of $Z_{n}$ exceeding $\zeta(3)$ this bound is correct, which is to say that $\log \operatorname{Pr}\left[Z_{n} \geq \zeta(3)+\varepsilon\right]=-\Theta(n)$. For example, the probability that every edge incident to vertex 1 has cost at least $1 / 2$ is $(1 / 2)^{n-1}$, and conditioned on this event, whp $Z_{n}=(1+o(1))(\zeta(3)+1 / 2)$. 
The behavior of the lower tail is not as simple to identify. A casual inspection may lead to the conjecture that the lower tail is even more tightly concentrated than the upper tail. The previous paragraph described how an overly large value of $Z_{n}$ can be "blamed" on a single vertex which has only expensive edges. However, for a single vertex to be similarly responsible for the cost of the tree being significantly lower than expected, it needs to have a lot of edges with cost less than $\zeta(3) / n$. This occurs with $\log$-probability of $-\Theta(n \log n)$.

The purpose of this note is to show that the lower tail of $Z_{n}$ is at least $e^{-C n}$ for any constant deviation less than $\zeta(3)$. (Note that, for example, $\operatorname{Pr}\left[Z_{n} \leq \zeta(3)-\left(\zeta(3)-n^{-10}\right)\right]$, is not at least $e^{-C n}$.)

Theorem 1 Let the random variable $Z_{n}$ be the cost of the minimum spanning tree when the edges of the complete graph $K_{n}$ have costs selected independently and uniformly at random in the interval $[0,1]$. Then, for any constant $\varepsilon<1$, there exists a constant $C_{\varepsilon}>0$, such that for all sufficiently large $n$,

$$
\operatorname{Pr}\left[Z_{n} \leq(1-\varepsilon) \zeta(3)\right] \geq e^{-C_{\varepsilon} n}
$$

This scaling behavior rules out the possibility that the lower tail of $Z_{n}$ is asymptotically more tightly concentrated than the currently best-known upper bound. This is in contrast with, for example, the result on the concentration of the eigenvalues of a random matrix due to Alon, Krivelevich, and $\mathrm{Vu}$ [2]. That paper considers how tightly an eigenvalue of a random matrix is concentrated around its mean, and shows that, for example, the log-probability of deviation of the first eigenvalue of the adjacency matrix of $\mathbb{G}_{n, 1 / 2}$ of scales like $-\Omega\left(n^{2}\right)$.

\section{Lower bound}

The argument establishing a lower bound is based on the observation that if the weights on the edges are independent and given by the minimum of 2 random variables selected uniformly at random from $[0,1]$ then the expected cost is $\zeta(3) / 2$ (this is proved by Steele in [10] and extended by Frieze and McDiarmid in [7]; in fact, the only feature of the edge weight distribution that is important to the expected value of $Z_{n}$ is the behavior of the density function at 0 .)

To make use of this observation, consider the following complicated way to generate $Z_{n}$ : Look first at a larger probability space, where each edge has 2 values, $X_{e}^{+}$and $X_{e}^{-}$, and each vertex has a polarity chosen uniformly at random, $\Phi(v) \in \pm 1$. Then, to obtain $Z_{n}$, consider the graph where edge $e=\{u, v\}$ has weight $Y_{e}=X_{e}^{\Phi(u) \Phi(v)}$.

Edge weights generated in this manner are identically distributed with the original model, and so the cost of the minimium spanning tree is distributed identically with $Z_{n}$. But with this generative procedure it is easy to obtain a lower bound on the log-probability of the event $\left\{Z_{n} \leq 3(\zeta(3)+\delta) / 4\right\}$ (when $\delta$ is arbitrarily small and $n$ is sufficiently large). Consider the minimum spanning tree in the graph where edge $e$ has weight $\min \left\{X_{e}^{+}, X_{e}^{-}\right\}$. Since this is a tree, there is a function $\psi$ which assigns every vertex a polarity so that $X_{e}^{\psi(u) \psi(v)}$ is the minimum of the 2 values. (To see this, designate some vertex to be the root, and start by 
arbitrarially assigning a polarity to the root, and then assigning the polarity of additional vertices in the order given by a breadth-first search of the minimum spanning tree.) If this function is the one that comes up, then the expected cost of $Z_{n}$ is asymptotic to $\zeta(3) / 2$, and, for sufficiently large $n$, by Markov's inequality, $\operatorname{Pr}\left[Z_{n} \geq 3 / 2(\zeta(3)+\delta) / 2 \mid \Phi=\psi\right] \leq 2 / 3$. The event $\{\Phi=\psi\}$ has the same probability as the event that $\Phi$ equals any other polarity function, so unconditionally, for sufficiently large $n, \operatorname{Pr}\left[Z_{n} \leq 3(\zeta(3)+\delta) / 4\right] \geq(1 / 3) 2^{-n}$.

For values of $\varepsilon \geq 1 / 4$, repeat this argument but with the larger probability space containing $k$ different weights for each edge, and vertex polarity chosen uniformly from the $k$

complex roots of unity, $\Phi(v) \in\left\{e^{2 \pi i \cdot \frac{0}{k}}, e^{2 \pi i \cdot \frac{1}{k}}, \ldots, e^{2 \pi i \cdot \frac{k-1}{k}}\right\}$. Again, considering as a weight the minimum of the $k$ weights on each edge leads to the expected value asymptotic to $\zeta(3) / k$, and, for sufficiently large $n$, the probability that this random variable exceeds $2(\zeta(3)+\delta) / k$ is at most $1 / 2$. Since there is again a function $\psi$ that results in selecting the minimum value for each edge in the minimum spanning tree, an upper-bound on the unconditional probability is

$$
\operatorname{Pr}\left[Z_{n} \leq 2(\zeta(3)+\delta) / k\right] \geq(1 / 2) k^{-n}
$$

Note that this argument also works when $k$ is a function of $n$, showing that

$$
\log \operatorname{Pr}\left[Z_{n}=\mathcal{O}(1 / k)\right]=-\Omega(n \log k)
$$

\section{Conclusion}

This note provides a simple proof that, for sufficiently large $n$, the probability of the cost of a minimum spanning tree being less than $(1-\varepsilon) \zeta(3)$ is at least $e^{-C_{\varepsilon} n}$. The proof technique described in Section 2 can also be applied to prove lower bounds on the probabilities of other functions being less than their means. It is only necessary to know that (1) when each variable is replaced by the minimum of $k$ copies, the expected value of the function decreases by a factor of $k$; and that (2) it is possible to describe which one of the $k$ copies is used by the function with $\mathcal{O}(n)$ bits. For the minimum perfect matching problem, it follows from the work of Aldous [1] that condition (1) is met, and condition (2) can be satisfied as above. For the minimum traveling salesperson problem, Wästlund's results in [11] show that condition (1) is met, and condition (2) can satisfied by setting polarities for $n-1$ vertices and specifying $n-1$ additional values for the edges incident to the vertex. For the minimum set of edges which can be partitioned into 2 disjoint spanning trees, condition (1) is implicit in the work of Avram and Bertsimas [3] (see [5] for additional detail), but it is not clear how to demonstrate condition (2) in a simple manner.

\section{References}

[1] Aldous, D. J. Asymptotics in the random assignment problem. Probab. Theory Related Fields 93, 4 (1992), 507-534. 
[2] Alon, N., Krivelevich, M., And Vu, V. H. On the concentration of eigenvalues of random symmetric matrices. Israel J. Math. 131 (2002), 259-267.

[3] Avram, F., and Bertsimas, D. The minimum spanning tree constant in geometrical probability and under the independent model: a unified approach. Ann. Appl. Probab. 2, 1 (1992), 113-130.

[4] Flaxman, A. D., Frieze, A., and Krivelevich, M. On the random 2-stage minimum spanning tree. Random Structures Algorithms 28, 1 (2006), 24-36.

[5] Flaxman, A. D., Vera, J., and Frieze, A. M. On edge-disjoint spanning trees in a randomly weighted complete graph. Manuscript in preparation, 2006.

[6] Frieze, A. M. On the value of a random minimum spanning tree problem. Discrete Appl. Math. 10, 1 (1985), 47-56.

[7] Frieze, A. M., And McDiarmid, C. J. H. On random minimum length spanning trees. Combinatorica 9, 4 (1989), 363-374.

[8] JAnson, S. The minimal spanning tree in a complete graph and a functional limit theorem for trees in a random graph. Random Structures Algorithms 7, 4 (1995), 337355.

[9] McDiarmid, C. On the method of bounded differences. In London Mathematical Society Lecture Note Series, vol. 141. Cambridge University Press, 1989, pp. 148-188.

[10] Steele, J. M. On Frieze's $\zeta(3)$ limit for lengths of minimal spanning trees. Discrete Appl. Math. 18, 1 (1987), 99-103.

[11] WÄstLund, J. The travelling salesman problem in the stochastic mean field model. Unpublished manuscript, 2006. 